\title{
NEDD8-activating enzyme inhibition induces cell cycle arrest and anaphase catastrophe in malignant T-cells
}

\author{
Adam S. Kittai ${ }^{1, *}$, Olga V. Danilova ${ }^{2, *}$, Vi Lam $^{2}$, Tingting Liu $^{2}$, Nur Bruss ${ }^{3}$, Scott \\ Best $^{3}$, Guang Fan ${ }^{3}$ and Alexey V. Danilov ${ }^{2}$ \\ ${ }^{1}$ The Ohio State University, Columbus, $\mathrm{OH} 43210$, USA \\ ${ }^{2}$ City of Hope Comprehensive Cancer Center, Duarte, CA 91010, USA \\ ${ }^{3}$ Oregon Health and Science University, Portland, OR 97239, USA \\ *These authors contributed equally to this work
}

Correspondence to: Alexey V. Danilov, email: adanilov@coh.org

Keywords: pevonedistat; T-cell lymphoma; chromosomal instability; anaphase catastrophe

Received: June 26, $2021 \quad$ Accepted: August 18, $2021 \quad$ Published: September 28, 2021

Copyright: $\odot 2021$ Kittai et al. This is an open access article distributed under the terms of the Creative Commons Attribution License (CC BY 3.0), which permits unrestricted use, distribution, and reproduction in any medium, provided the original author and source are credited.

\begin{abstract}
Peripheral T-cell lymphoma (PTCL) is characterized by poor outcomes. We and others have shown that targeting the NEDD8-activating enzyme (NAE) with an investigational inhibitor pevonedistat deregulates cell cycle and mitosis in lymphoma and leukemia. Here, we report that PTCL is characterized by increased rate of chromosomal instability. NAE inhibition promotes cell cycle arrest and induces multipolar anaphases in T-cell lymphoma cell lines, resulting in apoptosis, also observed in primary malignant PTCL cells treated with pevonedistat. We identified p2 $7^{\mathrm{Kip} 1}$ as a mediator of anaphase catastrophe in these cells. Targeting neddylation with pevonedistat may be a promising approach to treatment of PTCL.
\end{abstract}

\section{INTRODUCTION}

Peripheral T-cell lymphoma (PTCL) is an aggressive subset of non-Hodgkin lymphomas (NHL) characterized by poor outcomes [1]. Aberrant T-cell receptor and JAK-STAT signaling, genomic abnormalities targeting epigenetic modifiers and chromatin remodeling and altered cellular metabolism result in deregulated cell cycle, enhanced cell survival and proliferation in PTCL [2]. The substantial genetic and biologic heterogeneity [3] hinders success of targeted therapies in this disease and chemotherapy remains standard in both de novo and relapsed/refractory settings. Chromosomal instability (CIN) is predictive of poor outcomes in B-cell NHL [4] and other cancers and represents a tractable therapeutic target across multiple tumor types. We previously demonstrated that pharmacologic and genetic ablation of cyclin-dependent kinase 2 (CDK2) leads to an event termed anaphase catastrophe in lung cancer cells, confirmed with live-cell imaging [5-7]. This pathway preferentially eliminates aneuploid cancer cells by antagonizing clustering of supernumerary centrosomes during mitosis, forcing cells to undergo multipolar divisions [7]. However, it is not known if anaphase catastrophe occurs in NHL (PTCL) cells, and whether it may be induced by pharmacologic induction of endogenous CDK inhibitors in a cell.

Pevonedistat (MLN4924), an investigational small molecule inhibitor of the NEDD8-activating enzyme (NAE), demonstrated pre-clinical efficacy in B-cell NHL [8-11]. NAE ensures activation of the cullin-RING E3 ubiquitin ligases (CRLs) [12]. Pevonedistat forms a covalent adduct with NEDD8 (a ubiquitin-like modifier), resulting in NAE inhibition and accumulation of CRL substrates. CDT1, CDK inhibitors $\mathrm{p} 21^{\mathrm{Cip} 1} / \mathrm{p} 27^{\mathrm{Kip} 1}$ as well as checkpoint kinase Wee1 are among the CRL substrates which are increased following NAE inhibition. They are implicated in the anti-tumor effect of pevonedistat through induction of DNA damage, cell cycle arrest and apoptosis $[8,13,14]$.

Here we investigated the effect of NAE inhibition on CIN in malignant T-cells.

\section{RESULTS}

First, we quantified CIN in primary lymphoid tissues from patients with PTCL. Abnormal anaphases were detected with the frequency of $56.1 \% \pm 2.9 \%$ (Figure 1A). 
All tumors surveyed in this study $(N=14)$ demonstrated high level of mis-segregation using the cutoff previously established in diffuse large B-cell lymphoma (DLBCL; $31.1 \%$ ) [4]. Lagging chromosomes and chromatin bridges were detected in $24.8 \pm 2.9 \%$ and $31.2 \pm 2.9 \%$ of anaphase cells, respectively (Figure 1A and 1B). Abnormal anaphases were not observed in reactive lymph nodes $(N=5)$. Thus, PTCL tumors exhibited profound CIN as assessed by chromosomal mis-segregation events, which exceeded that observed in DLBCL.

Next, we evaluated the effect of NAE inhibition on survival and proliferation of malignant T-cells. Pevonedistat induced apoptosis of primary PTCL cells after a 24-hour exposure (Figure 2A). Pevonedistat exposure diminished proliferation of SR, Jurkat, SUP-T1 and HuT-78 cells (Figure 2B). While minimal cell line apoptosis was seen at 24 hours, 48 -hour exposure to pevonedistat induced apoptosis with an $\mathrm{IC}_{50} \sim 0.3$ $\mu \mathrm{M}$ (Figure 2C). Meanwhile, HH cells were resistant. Treatment with pevonedistat disrupted cullin neddylation in a dose-dependent manner (Figure 2D). We observed rapid increases of CRL substrate proteins, including CDT1, p2 $1^{\text {Cipl }}$ and $\mathrm{p} 27^{\text {Kipl }}$ (hereinafter p27) following NAE inhibition. HH cells and primary PTCL cells showed weak induction of CDT1, consistent with their low proliferation rate (Figure 2E). Pevonedistat induced DNA damage as evidenced by an increase in $\gamma \mathrm{H} 2 \mathrm{AX}$ (Figure 2D). SR and Jurkat cells, but not HH cells, exhibited arrest in S and $\mathrm{G}_{2} / \mathrm{M}$ phases of cell cycle when treated with pevonedistat (Figure 3).

Since p27 (an endogenous CDK inhibitor) accumulated in malignant T-cells treated with pevonedistat, we studied anaphase catastrophe in this setting. We observed a significant induction of anaphase catastrophe in SR and Jurkat cells, but not HH cells, following NAE inhibition (Figure 4A). Upon 24-hour treatment with $0.25 \mu \mathrm{M}$ pevonedistat, $36.0 \pm 5.0 \%$ of Jurkat and $23.3 \pm 4.4 \%$ of SR cells exhibited multipolar anaphases, compared with $6.0 \pm 3.1 \%$ and $2.0 \pm 0.7 \%$ with vehicle control, respectively. Thus, induction of anaphase catastrophe preceded apoptosis in those cells.

To evaluate whether induction of p27 is involved in mitotic deregulation in this context, we employed CRISPR-Cas9 editing. Introduction of $C D K N 1 B$ gRNA resulted in complete loss of p27 protein in both Jurkat and SR cell lines (Figure 4B). p27-deficient cells continued to exhibit arrest in $S / \mathrm{G}_{2} / \mathrm{M}$ phases of cell cycle (Figure $4 \mathrm{C}$ ), suggesting that this effect is likely Cdt1-mediated as previously shown in other model systems $[8,15]$. However, SR and Jurkat cells lacking p27 no longer exhibited anaphase catastrophe following treatment with pevonedistat (Figure 4D). Furthermore, p27-deficient cells demonstrated slightly diminished susceptibility to pevonedistat-induced apoptosis (Figure 4E). This indicates that p27 accumulation following NAE inhibition contributes to anaphase catastrophe and may in part mediate apoptosis in malignant T-cells.

\section{DISCUSSION}

While we previously reported that lung cancer cells undergo anaphase catastrophe when exposed to chemotherapy and CDK2 inhibitors [5, 6], here we show that this pathway is also induced in neoplastic T-cells.
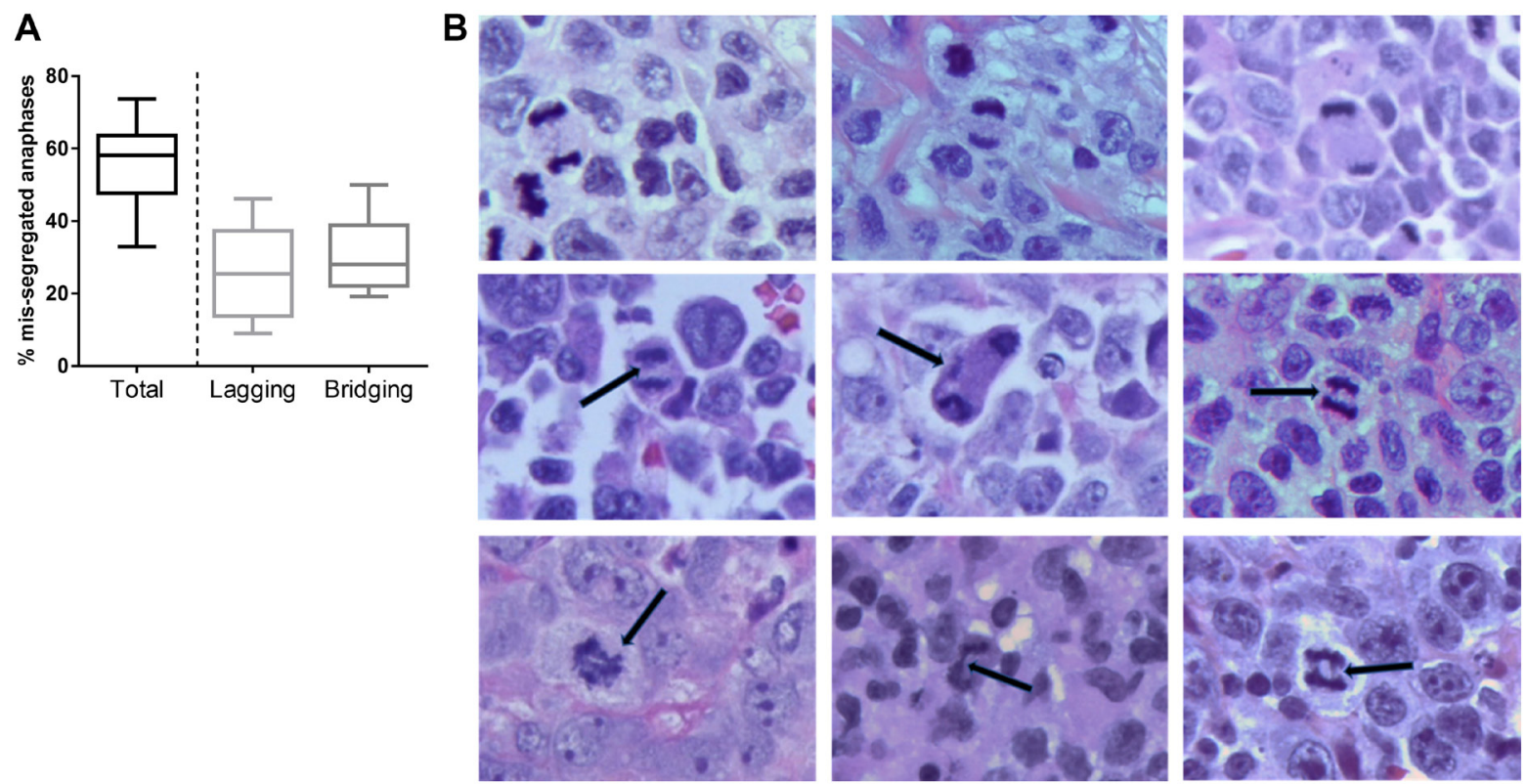

Figure 1: Chromosomal instability in PTCL. (A) A box-and-whiskers graph showing frequency of cells which exhibit mis-segregated chromosomes during anaphase (median, 25th/75th quartiles, min/max). (B) Images of H\&E-stained T-cell lymphoma tissues showing anaphase. Representative examples of normal anaphase (top row), anaphase with lagging chromosomes (middle row), and chromatin bridges (bottom row) are shown. Scale bar, $5 \mu \mathrm{m}$. 
A

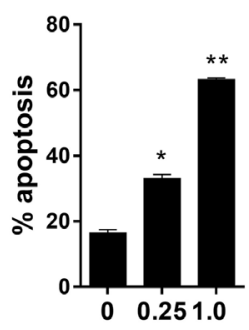

Pevonedistat $(\mu \mathrm{M})$
B $\square 0.05 \mu \mathrm{M}$

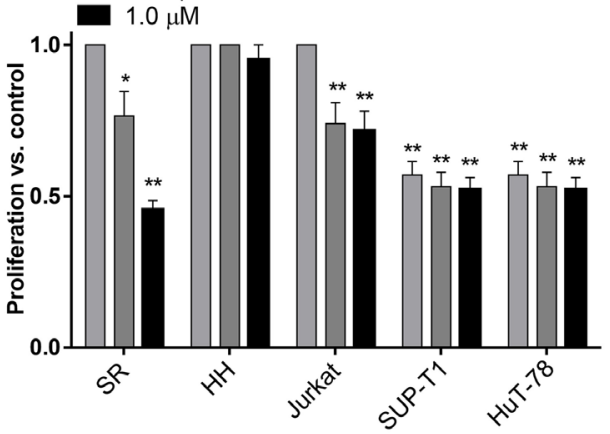

C
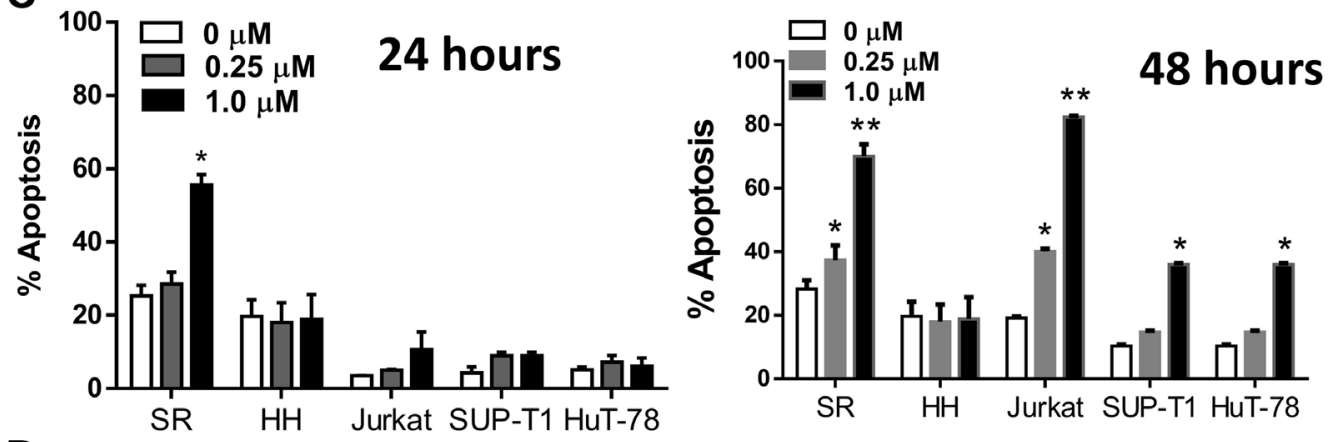

D

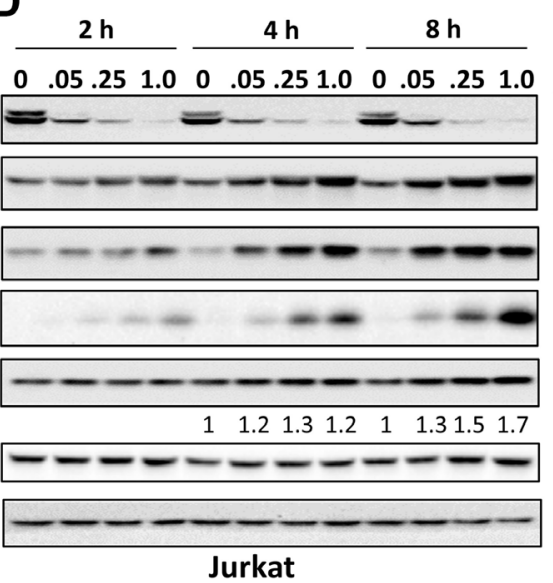

$\frac{2 h}{0.251 .0} \frac{4 h}{0.251 .0} \frac{8 h}{0 \quad 251.0} \frac{24 h}{0.251 .0 \text { Pevo, } \mu M}$
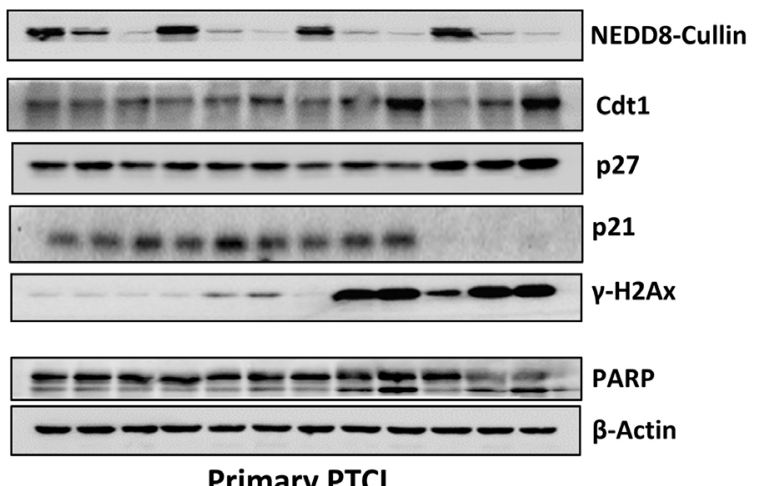

$2 \mathrm{~h}-4 \mathrm{~h}-8 \mathrm{~h}$

$\begin{array}{lllllllllllll}0 & .05 .25 & 1.0 & 0 & .05 & .25 & 1.0 & 0 & .05 & .25 & 1.0 & \text { Pevo, } \mu \mathrm{M}\end{array}$

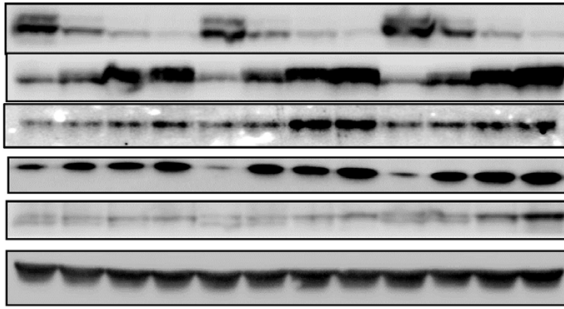

NEDD8-Cullin

Cdt1

p27

$\mathrm{V}-\mathrm{H} 2 \mathrm{Ax}$

Cleaved PARP

$\beta$-Actin

SR

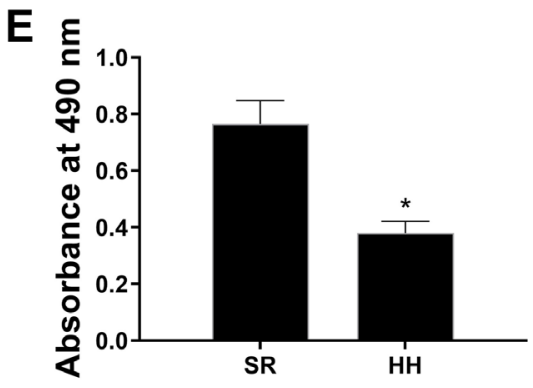

Figure 2: Pevonedistat induces neoplastic T cell apoptosis. (A) Circulating tumor cells from 3 individual patients with PTCL were treated with pevonedistat for 48 hours and assayed for apoptosis (in duplicates) in the CD3 ${ }^{+}$population. (B) Cells were incubated with the indicated concentrations of pevonedistat or vehicle control for 72 hours ( 6 wells per condition; performed in triplicates). Cellular proliferation was measured in a tetrazolium-based colorimetric assay. Values are normalized to vehicle-treated control. Data are the mean \pm SEM. (C) Cells were treated with the indicated concentrations of pevonedistat for 24 (left) or 48 hours (right). Apoptosis was determined by Annexin-V staining. Data are mean \pm SEM. (D) Jurkat, SR and primary PTCL cells were treated with pevonedistat as indicated, proteins were lysed and subjected to immunoblotting. (E) Cells were seeded at equal density, cultured for 72 hours and proliferation was measured in a tetrazolium-based colorimetric assay. ${ }^{*} p<0.05$ and ${ }^{* *} p<0.01$ vs. control. 
p27 complexes with CDK2-Cyclin E/A, thus restraining progression through $\mathrm{G}_{1} / \mathrm{S}$ phase of the cell cycle [16]. We now also demonstrate an additional role, where following NAE inhibition p27 mediates anaphase catastrophe. We did not explore whether this effect is mediated via attenuated activity of $\mathrm{CDK} 2$. It has been recognized that in addition to CDK2-Cyclin E/A, p27 has complex interplay with other CDK complexes, including CDK1 and CDK4/6 [16, 17]. While loss of CDK1 is also known to mediate anaphase catastrophe, potential role of CDK4/6 in this setting has not been explored [6]. Furthermore, we have not investigated other CRL substrates which could potentially be involved in this mechanism, such as $\mathrm{p} 21^{\mathrm{Cip} 1}$, which can attenuate activity of multiple CDK complexes, or proteins involved in regulation of DNA damage checkpoint, i.e. CDT1 and Wee1. Future studies should also determine if pevonedistat would synergize with chemotherapy to induce anaphase catastrophe.

In sum, we demonstrate that PTCL exhibits pronounced chromosomal mis-segregation. Targeting NAE with pevonedistat leads to cell cycle arrest and anaphase catastrophe in neoplastic T-cells. This effect, possibly coupled with immunomodulatory activity of pevonedistat recently described by our group [18], justifies continued exploration of pevonedistat as a novel therapeutic approach in T-cell NHL.

\section{MATERIALS AND METHODS}

\section{Cells, cell cycle and apoptosis}

Following approval by the IRB, PTCL cells were isolated from PBMC of three patients with high circulating tumor burden using standard Ficoll-Hypaque technique ( $>95 \% \mathrm{CD}^{+} / \mathrm{CD}^{-}$tumor cells). SR, Jurkat and SUP-T1 (T-lymphoblast) cell lines and Sezary HH and HuT-78 cells were obtained from the American Type Culture Collection (ATCC). All cells were cultured in RPMI-1640 medium supplemented with $15 \%$ fetal bovine serum.

To measure cell proliferation, cells were plated in 96-well plates at 3000/well in $100 \mu \mathrm{L}$ (6 per sample) and incubated for 72 hours. Viable cells were measured using

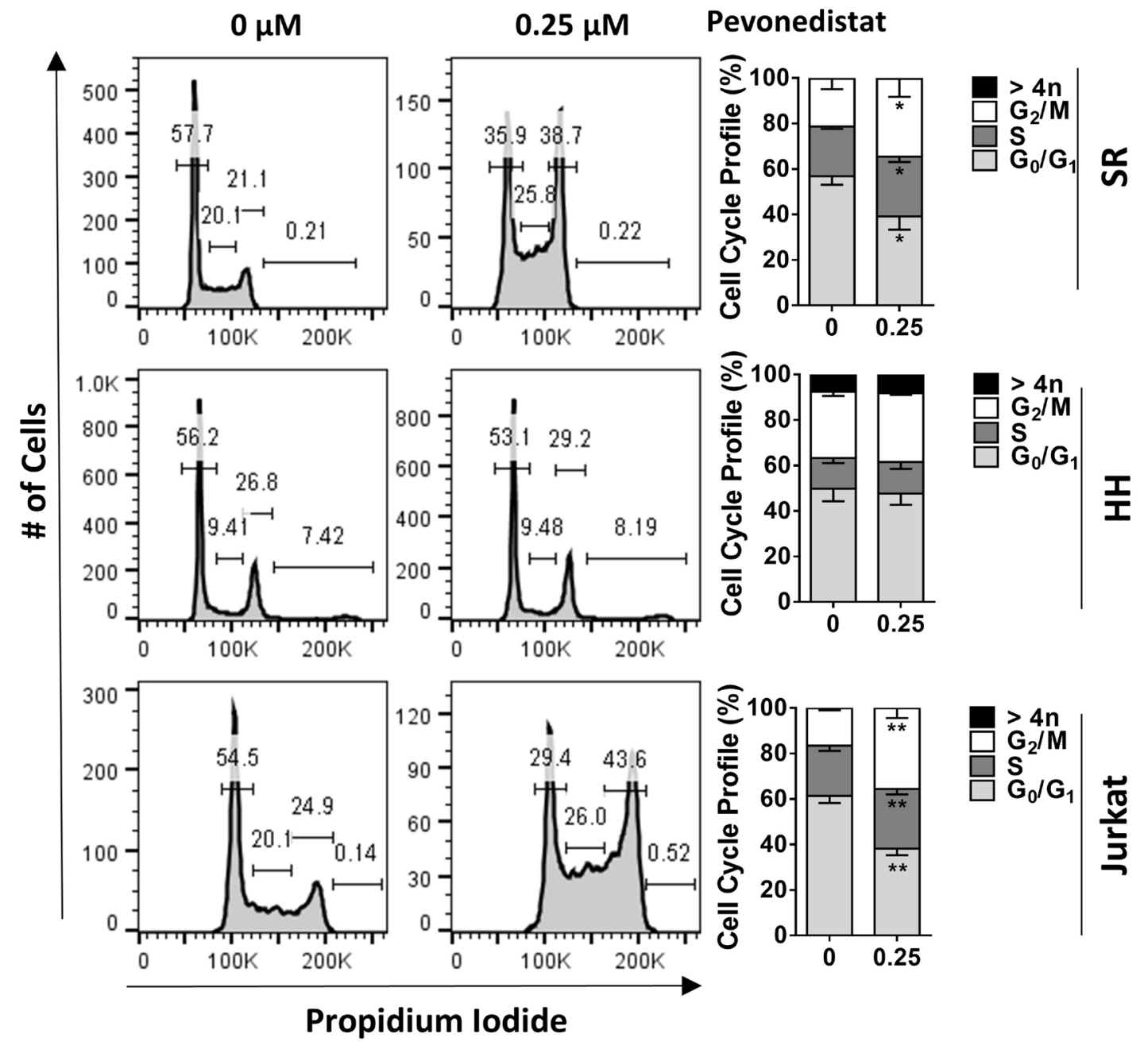

Figure 3: Pevonedistat induces cell cycle arrest. Cells were treated with $0.25 \mu \mathrm{M}$ pevonedistat for 24 hours and assayed for cell cycle profiling using propidium iodide staining. ${ }^{*} p<0.05,{ }^{* *} p<0.01$ vs. control. 
a CellTiter Aqueous One Solution Cell Proliferation Assay (Promega).

Cell apoptosis was measured in duplicates using the ApoScreen Annexin V Apoptosis Kit as previously described (Southern Biotech) [8]. For cell cycle analysis, 2 $\times 10^{5}$ cells were fixed in ice cold $70 \%$ ethanol while being vortexed, incubated on ice for 15 minutes, washed in PBS and resuspended in $250 \mu$ l of staining solution containing $20 \mathrm{ng} / \mathrm{ml}$ propidium iodide, $200 \mathrm{ng} / \mathrm{ml}$ RNAse A (Sigma Aldrich), 0.1\% Triton-X 100 and $1 \mu$ CD19-FITC mAb in PBS. Cells were incubated for 15 minutes and submitted to flow cytometry. Analysis was performed using FlowJo software (Tree Star).

Pevonedistat was provided by Millennium
A

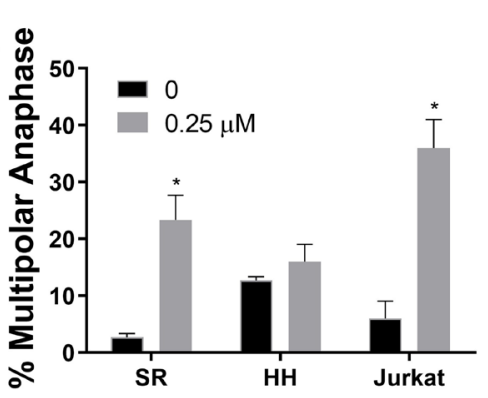

SR
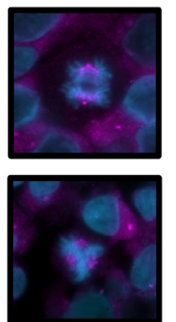

$\mathrm{HH}$

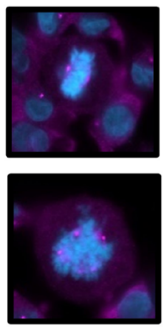

Jurkat

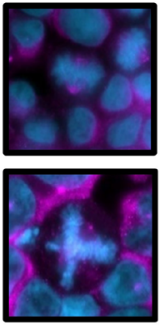

Control

$0.25 \mu \mathrm{M}$

Pevonedistat
B

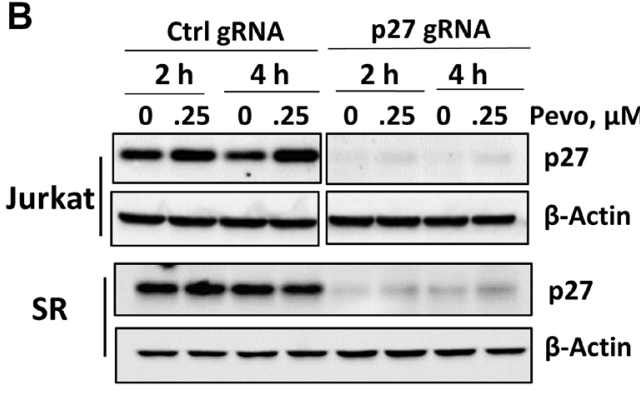

D

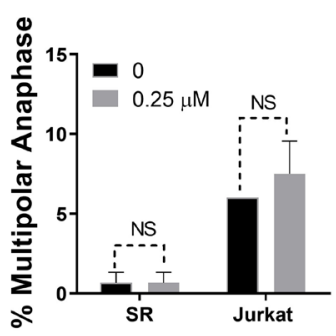

E

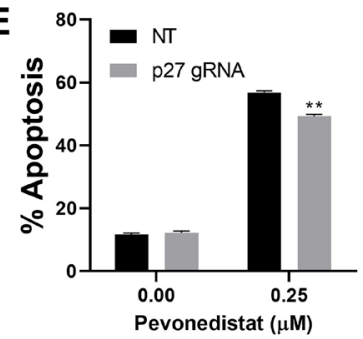

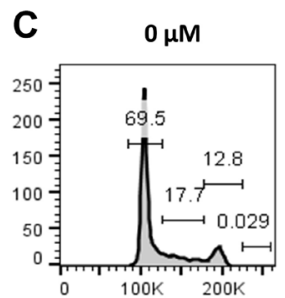
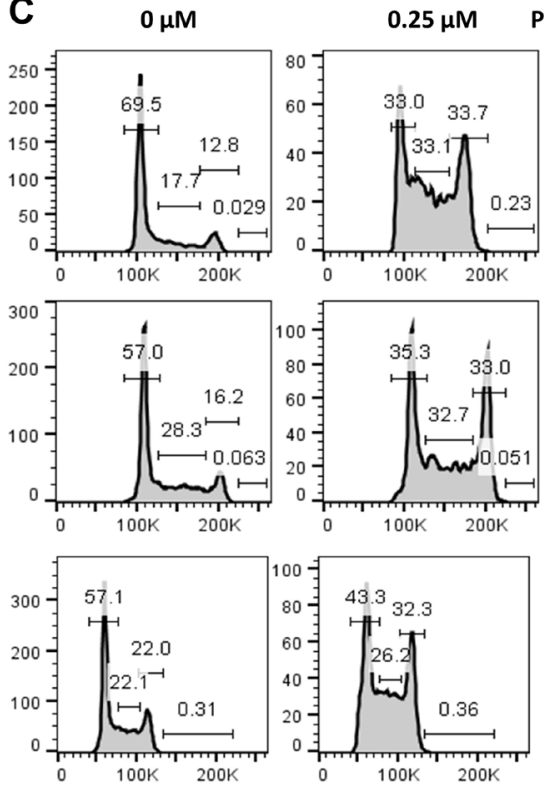

Pevonedistat
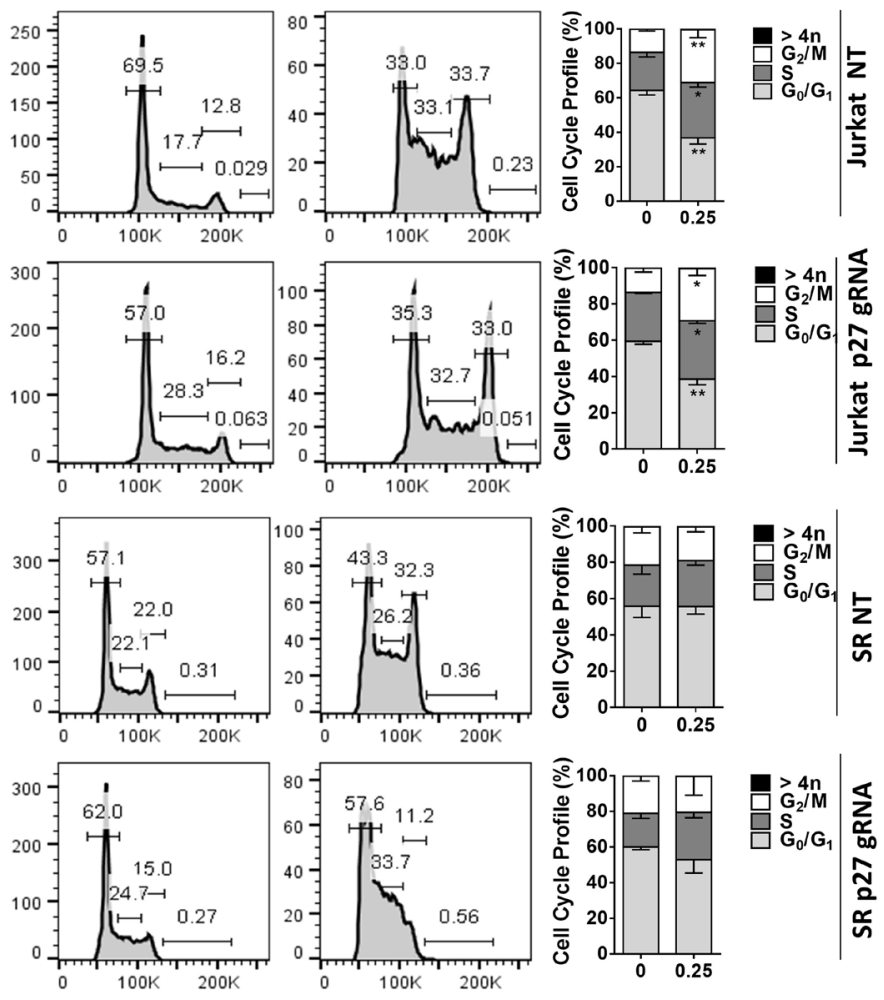

Figure 4: Malignant T-cells undergo anaphase catastrophe following NAE inhibition. (A) Cells were incubated with the indicated concentration of pevonedistat or vehicle control for 24 hours. Cells were immunostained with $\gamma$-tubulin (pink) and counterstained with DAPI (blue). Total anaphases were counted (50) and the proportion of multipolar anaphases is shown. Data are the mean \pm SEM of at least 3 independent experiments. Representative examples of normal mitosis and multipolar anaphase are shown. (B) Jurkat and SR cells were transfected with a lentiviral CRISPR toolbox to achieve knockout of p27, or vector control. Cells were treated with pevonedistat as indicated, proteins were lysed and subjected to immunoblotting. (C) Cells were treated with pevonedistat for 24 hours and subjected to cell cycle profiling with propidium iodide staining. A summary of three independent experiments is shown. (D) p27-deficient cells were incubated with $0.25 \mu \mathrm{M}$ pevonedistat or vehicle control for 24 hours. Cells were immunostained with $\gamma$-tubulin and counterstained with DAPI. Total anaphases were counted (50) and the proportion of multipolar anaphases is shown. Data are the mean \pm SEM of six (Jurkat) and three (SR) independent experiments. (E) Jurkat cells were treated with $0.25 \mu \mathrm{M}$ pevonedistat or vehicle control for 48 hours. Apoptosis was determined by Annexin-V staining. Data are mean \pm SEM of 6 independent experiments. ${ }^{*} p<0.05$ and ${ }^{* *} p<0.01$ vs. control. 
Pharmaceuticals, Inc. (Cambridge, MA, USA), a wholly owned subsidiary of Takeda Pharmaceutical Company Limited.

\section{Immunoblotting}

Cells were lysed in RIPA buffer with supplements and proteins were analyzed by immunoblotting as previously described [8]. The following antibodies were used: NEDD8, p21 $1^{\text {Cip1 }}$, p27 ${ }^{\text {Kip1 } 1}$ CDT1, $\gamma$-H2AX, PARP and cleaved PARP, $\beta$-Actin, and horseradish peroxidaseconjugated anti-mouse and anti-rabbit antibodies (Cell Signaling Technologies). In each case, a representative image of at least 3 independent immunoblotting experiments is shown.

\section{CRISPR-Cas9 genome editing}

A lentiCRISPRv2 system (Addgene, 62988) was used as described in GeCKO lentiviral CRISPR toolbox [19]. Lentivirus was packaged using psPAX2 (Addgene \#12260) and VSVG (Thermo Fisher, K497500) plasmids in HEK293T cells (ATCC) using jetPRIME transfection reagent (Polyplus Transfection). Viral supernatants were collected at 48 and 60 hours and quantified with qPCR Lentivirus Titration Kit (Applied Biological Materials Inc). Lentivirus was transduced in $1 \times 10^{6}$ Jurkat and $\mathrm{HH}$ cells at a MOI of 10 using spinoculation with 2500 $\mathrm{RPM}$ for $90 \mathrm{~min}$ at $30^{\circ} \mathrm{C}$. Infected cells were selected in $2 \mu \mathrm{g} / \mathrm{mL}$ puromycin (Gibco) 2 days post transduction. CDKN1B (p27 ${ }^{\text {Kipl1 }}$ ) gRNA oligos: forward 5'-CACCG CAGGAACCTCTTCGGCCCGG(TGG)-3', reverse 5'-AAACCCGGGCCGAAGAGGTTCCTGC-3'.

\section{Anaphase catastrophe}

Cells were fixed in 10\% formalin, stained with anti- $\gamma$-tubulin-specific antibody (Thermo Fisher), and independently mounted with Pro-Long Gold antifade reagent supplemented with 40,6-diamidino-2-phenylindole (DAPI; Thermo Fisher). Fluorescent images were captured with an F-view II monochrome camera (Olympus, U-CMAD3) mounted on Zeiss Apotome 2. Total anaphase cells were counted and those that contained $\geq 3$ spindle poles were scored as multipolar.

\section{Chromosomal mis-segregation in lymphoid tissues}

Fourteen patients with PTCL (6 anaplastic large cell lymphoma, 3 angioimmunoblastic T-cell lymphoma, one NK/T-cell lymphoma and 4 PTCL NOS) were included in the study per the local IRB regulations. Formalin-fixed paraffin-embedded samples were stained with H\&E. Cells undergoing anaphase were surveyed for evidence of chromosome mis-segregation as previously reported
[4]. An average of 59 (range, 20 to 100) anaphases were scored per sample. Normal anaphase was defined by the absence of any chromatin staining between the chromosome masses, while mis-segregation was defined by the existence of either lagging chromosomes (an area of hematoxylin staining completely isolated in between the remaining segregating chromosomes during anaphase) or chromatin bridges (at least one continuous band of hematoxylin staining linking the segregating chromosomes).

\section{Statistical analysis}

Statistical analysis was performed with Student $t$ test in GraphPad Prism software. ${ }^{*} p<0.05$ and ${ }^{* *} p<$ 0.01 throughout the manuscript. All experiments were performed at least in biological triplicates.

\section{Author contributions}

A.S.K. and A.V.D. designed the study. A.S.K., V.L., S.B., N.B., T.L., G.F. and O.V.D. performed research and analyzed data. A.S.K. and A.V.D. wrote the manuscript. All authors participated in drafting, revising, and approving the final manuscript.

\section{ACKNOWLEDGMENTS}

A.S.K. was supported by the American Society of Hematology Research Training Award for Fellows. A.V.D. was supported in part by the Leukemia \& Lymphoma Society Scholar in Clinical Research Award \#2319-19 and the American Society of Hematology Bridge Grant Award. We acknowledge expert technical assistance by staff in the Advanced Multiscale Microscopy Shared Resource, supported by the OHSU Knight Cancer Institute (NIH P30 CA069533).

\section{CONFLICTS OF INTEREST}

A.S.K. consults for Bristol Meyers Squibb. A.V.D. received consulting fees from Abbvie, AstraZeneca, BeiGene, Genentech, Pharmacyclics and TG Therapeutics and has ongoing research funding from AstraZeneca, Bayer Oncology, Bristol Meyers Squibb, Genentech, MEI Pharma, SecuraBio, TG Therapeutics and Takeda Oncology.

\section{REFERENCES}

1. Bellei M, Foss FM, Shustov AR, Horwitz SM, Marcheselli L, Kim WS, Cabrera ME, Dlouhy I, Nagler A, Advani RH, Pesce EA, Ko YH, Martinez V, et al, and International T-cell Project Network. The outcome of peripheral T-cell lymphoma patients failing first-line therapy: 
a report from the prospective, International T-Cell Project. Haematologica. 2018; 103:1191-97. https://doi. org/10.3324/haematol.2017.186577. [PubMed]

2. Van Arnam JS, Lim MS, Elenitoba-Johnson KSJ. Novel insights into the pathogenesis of T-cell lymphomas. Blood. 2018; 131:2320-30. https://doi.org/10.1182/ blood-2017-11-764357. [PubMed]

3. Watatani Y, Sato Y, Miyoshi H, Sakamoto K, Nishida K, Gion Y, Nagata Y, Shiraishi Y, Chiba K, Tanaka H, Zhao L, Ochi Y, Takeuchi Y, et al. Molecular heterogeneity in peripheral T-cell lymphoma, not otherwise specified revealed by comprehensive genetic profiling. Leukemia. 2019; 33:2867-83. https://doi.org/10.1038/s41375-0190473-1. [PubMed]

4. Bakhoum SF, Danilova OV, Kaur P, Levy NB, Compton DA. Chromosomal instability substantiates poor prognosis in patients with diffuse large B-cell lymphoma. Clin Cancer Res. 2011; 17:7704-11. https://doi.org/10.1158/1078-0432. CCR-11-2049. [PubMed]

5. Hu S, Danilov AV, Godek K, Orr B, Tafe LJ, RodriguezCanales J, Behrens C, Mino B, Moran CA, Memoli VA, Mustachio LM, Galimberti F, Ravi S, et al. CDK2 Inhibition Causes Anaphase Catastrophe in Lung Cancer through the Centrosomal Protein CP110. Cancer Res. 2015; 75:202938. https://doi.org/10.1158/0008-5472.CAN-14-1494. [PubMed]

6. Danilov AV, Hu S, Orr B, Godek K, Mustachio LM, Sekula D, Liu X, Kawakami M, Johnson FM, Compton DA, Freemantle SJ, Dmitrovsky E. Dinaciclib Induces Anaphase Catastrophe in Lung Cancer Cells via Inhibition of CyclinDependent Kinases 1 and 2. Mol Cancer Ther. 2016; 15:2758-66. https://doi.org/10.1158/1535-7163.MCT-160127. [PubMed]

7. Kawakami M, Mustachio LM, Liu X, Dmitrovsky E. Engaging Anaphase Catastrophe Mechanisms to Eradicate Aneuploid Cancers. Mol Cancer Ther. 2018; 17:72431. https://doi.org/10.1158/1535-7163.MCT-17-1108. [PubMed]

8. Paiva C, Godbersen JC, Berger A, Brown JR, Danilov AV. Targeting neddylation induces DNA damage and checkpoint activation and sensitizes chronic lymphocytic leukemia B cells to alkylating agents. Cell Death Dis. 2015; 6:e1807. https://doi.org/10.1038/cddis.2015.161. [PubMed]

9. Milhollen MA, Traore T, Adams-Duffy J, Thomas MP, Berger AJ, Dang L, Dick LR, Garnsey JJ, Koenig E, Langston SP, Manfredi M, Narayanan U, Rolfe M, et al. MLN4924, a NEDD8-activating enzyme inhibitor, is active in diffuse large B-cell lymphoma models: rationale for treatment of NF-\{kappa\}B-dependent lymphoma. Blood. 2010; 116:1515-23. https://doi.org/10.1182/ blood-2010-03-272567. [PubMed]

10. Czuczman NM, Barth MJ, Gu J, Neppalli V, Mavis C, Frys SE, Hu Q, Liu S, Klener P, Vockova P, Czuczman MS, Hernandez-Ilizaliturri FJ. Pevonedistat, a NEDD8activating enzyme inhibitor, is active in mantle cell lymphoma and enhances rituximab activity in vivo. Blood. 2016; 127:1128-37. https://doi.org/10.1182/ blood-2015-04-640920. [PubMed]

11. Torka P, Mavis C, Kothari S, Belliotti S, Gu J, Sundaram S, Barth M, Hernandez-Ilizaliturri FJ. Pevonedistat, a NEDD8-Activating Enzyme Inhibitor, Induces Apoptosis and Augments Efficacy of Chemotherapy and Small Molecule Inhibitors in Pre-clinical Models of Diffuse Large B-cell Lymphoma. EJHaem. 2020; 1:122-32. https://doi. org/10.1002/jha2.2. [PubMed]

12. Chiba T, Tanaka K. Cullin-based ubiquitin ligase and its control by NEDD8-conjugating system. Curr Protein Pept Sci. 2004; 5:177-84. https://doi. org/10.2174/1389203043379783. [PubMed]

13. Milhollen MA, Narayanan U, Soucy TA, Veiby PO, Smith PG, Amidon B. Inhibition of NEDD8-activating enzyme induces rereplication and apoptosis in human tumor cells consistent with deregulating CDT1 turnover. Cancer Res. 2011; 71:3042-51. https://doi.org/10.1158/0008-5472. CAN-10-2122. [ [PubMed]

14. Mackintosh C, Garcia-Dominguez DJ, Ordonez JL, GinelPicardo A, Smith PG, Sacristan MP, de Alava E. WEE1 accumulation and deregulation of S-phase proteins mediate MLN4924 potent inhibitory effect on Ewing sarcoma cells. Oncogene. 2013; 32:1441-51. https://doi.org/10.1038/ onc.2012.153. [PubMed]

15. Lin JJ, Milhollen MA, Smith PG, Narayanan U, Dutta A. NEDD8-targeting drug MLN4924 elicits DNA rereplication by stabilizing Cdt1 in $\mathrm{S}$ phase, triggering checkpoint activation, apoptosis, and senescence in cancer cells. Cancer Res. 2010; 70:10310-20. https://doi.org/10.1158/00085472.CAN-10-2062. [PubMed]

16. Bencivenga D, Caldarelli I, Stampone E, Mancini FP, Balestrieri ML, Della Ragione F, Borriello A. p27 ${ }^{\text {Kip1 }}$ and human cancers: A reappraisal of a still enigmatic protein. Cancer Lett. 2017; 403:354-65. https://doi.org/10.1016/j. canlet.2017.06.031. [PubMed]

17. Martin A, Odajima J, Hunt SL, Dubus P, Ortega S, Malumbres M, Barbacid M. Cdk2 is dispensable for cell cycle inhibition and tumor suppression mediated by p27(Kip1) and p21(Cip1). Cancer Cell. 2005; 7:591-98. https://doi.org/10.1016/j.ccr.2005.05.006. [PubMed]

18. Best S, Lam V, Liu T, Bruss N, Kittai A, Danilova OV, Murray S, Berger A, Pennock ND, Lind EF, Danilov AV. Immunomodulatory effects of pevonedistat, a NEDD8activating enzyme inhibitor, in chronic lymphocytic leukemia-derived T cells. Leukemia. 2021; 35:156-68. https://doi.org/10.1038/s41375-020-0794-0. [PubMed]

19. Shalem O, Sanjana NE, Hartenian E, Shi X, Scott DA, Mikkelson T, Heckl D, Ebert BL, Root DE, Doench JG, Zhang F. Genome-scale CRISPR-Cas9 knockout screening in human cells. Science. 2014; 343:84-87. https://doi. org/10.1126/science.1247005. [PubMed] 